Homology, Homotopy and Applications, vol.6(1), 2004, pp.283-298

\title{
GALOIS THEORY AND DOUBLE CENTRAL EXTENSIONS
}

\author{
MARINO GRAN AND VALENTINA ROSSI \\ (communicated by George Janelidze)
}

\begin{abstract}
We define a Galois structure between central extensions and extensions in a Maltsev variety. By using the theory of commutators we introduce double central extensions. We then prove that the covering morphisms relative to this Galois structure are precisely the double central extensions.
\end{abstract}

\section{Introduction}

The adjunction between the categories Grp of groups and $A b$ of abelian groups

$$
A b \underset{\text { inclusion }}{\stackrel{\text { abelianization }}{\perp}} \operatorname{Grp}
$$

gives rise to a natural Galois structure in the sense of the categorical Galois theory of Janelidze [3]. This Galois structure $\Gamma$ determines a class of homomorphisms, called $\Gamma$-coverings, which turn out to be exactly the central extensions of groups, i.e. the surjective homomorphisms of groups with kernel contained in the centre of their domain. Accordingly, the theory of the central extensions is a special case of the categorical Galois theory.

This correspondence between $\Gamma$-coverings and central extensions still holds in any Maltsev variety [5], i.e. in any variety of universal algebras whose theory has a ternary term $p(x, y, z)$ such that $p(x, y, y)=x$ and $p(x, x, y)=y$. The main tool allowing this generalization to Maltsev varieties comes from the fact that, in this context, there is a well-behaved theory of commutators [9] extending the notion of commutator for normal subgroups in the category of groups.

In [4], another Galois structure $\Gamma$ was defined starting from the adjunction between extensions (i.e. surjective homomorphisms of groups) and central extensions:

$$
\operatorname{Centr}(\operatorname{Grp}) \underset{U}{\stackrel{F}{\stackrel{F}{\perp}}} \operatorname{Ext}(\operatorname{Grp}) .
$$

The left adjoint $F: \operatorname{Ext}(\operatorname{Grp}) \rightarrow \operatorname{Centr}(\operatorname{Grp})$ sends an extension $f: A \rightarrow B$ to the central extension $F(f): \frac{A}{[K[f], A]} \rightarrow B$, where $K[f]$ is the kernel of $f$ and $[K[f], A]$ is

The authors would like to acknowledge financial support from the Università degli Studi di Udine and the Université du Littoral Côte d'Opale.

Received May 8, 2004, revised June 16, 2004; published on August 6, 2004.

2000 Mathematics Subject Classification: 08B05, 18G50, 13B05.

Key words and phrases: Categorical Galois theory, Maltsev varieties, commutators, double central extensions.

(c) 2004, Marino Gran and Valentina Rossi. Permission to copy for private use granted. 
the usual commutator of subgroups. The $\Gamma$-coverings relative to this second Galois structure also admit an elegant description in terms of the group-theoretic commutator, which defines the double central extensions of groups [4].

In the present article we first define a Galois structure between the extensions $\operatorname{Ext}(\mathcal{V})$ and the central extensions $\operatorname{Centr}(\mathcal{V})$ in any Maltsev variety $\mathcal{V}$, which becomes the one defined by Janelidze when $\mathcal{V}=\operatorname{Grp}$. We then characterize the $\Gamma$ coverings relative to this Galois structure in terms of two commutator conditions. The equivalence of $\Gamma$-coverings and double central extensions gives to this last notion its full meaning in any Maltsev variety $\mathcal{V}$.

The paper is divided into the following sections:

1. Maltsev varieties and commutators

2. Galois structures

3. Galois structure of central extensions

4. Double central extensions

5. The characterization of $\Gamma$-coverings

In the first section we recall the definition of the commutator in a Maltsev variety and some of its basic properties. In Section 2 we recall the basic notions of categorical Galois theory. In Section 3 we construct a particular Galois structure $\Gamma$ between extensions and central extensions in a Maltsev variety, and we give a simplified definition of $\Gamma$-covering. In Section 4 we define the double central extensions and show that any $\Gamma$-covering is a double central extension. In the last section, thanks to a method similar to the one used by Janelidze and Kelly in [5], we show the equivalence of the notions of $\Gamma$-covering and double central extension.

\section{Maltsev varieties and commutators}

In this first section we briefly recall some basic properties of commutators in Maltsev varieties. We adopt the categorical approach developed by Janelidze and Pedicchio in [6]. We shall follow the presentation given by Janelidze and Kelly in [5].

In the following $\mathcal{V}$ will denote a fixed variety of universal algebras. Let $\mathcal{V}$ be determined by a fixed set of operators $\Omega=\Omega_{0} \cup \Omega_{1} \cup \Omega_{2} \ldots$ and a certain set of identities. A variety $\mathcal{V}$ is Maltsev $[\mathbf{9}]$ if it has a ternary (possibly derived) operation $p(x, y, z)$ satisfying the identities

$$
p(x, y, y)=x \quad \text { and } \quad p(x, x, y)=y .
$$

Among the examples of Maltsev varieties there are groups, where a Maltsev operation is given by $p(x, y, z)=x \cdot y^{-1} \cdot z$, abelian groups, quasigroups, rings, Lie algebras, Heyting algebras and crossed modules.

Let us recall a classical result on Maltsev varieties $[\mathbf{8}],[\mathbf{9}]$ :

Theorem 1.1. Let $\mathcal{V}$ be a variety. Then the following conditions are equivalent:

1. $\mathcal{V}$ is a Maltsev variety;

2. any reflexive homomorphic relation in $\mathcal{V}$ is a congruence;

3. for any congruences $R$ and $S$ on any algebra $A, R \vee S=R \circ S$. 
The following characterization of Maltsev varieties will be also needed:

Proposition 1.2. [2] Let $\mathcal{V}$ be a variety. Then $\mathcal{V}$ is a Maltsev variety if and only if the following property holds: given a pushout of surjective homomorphisms $r$ and $s$

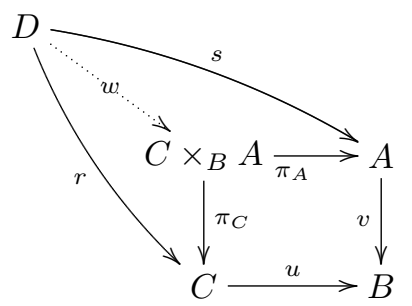

the comparison arrow $w$ to the pullback of $u$ and $v$ is a surjective homomorphism.

Any Maltsev variety is congruence modular. This means that the lattice of congruences on any algebra $A$ in $\mathcal{V}$ is modular, i.e. $R \wedge(S \vee T)=(R \wedge S) \vee T$ for any congruences $R, S$ and $T$ on $A$ such that $T \leqslant R$.

Maltsev varieties provide a very rich context in which one can develop the theory of commutators of congruences. We shall adopt the definition of the commutator given in $[\mathbf{5}]$ ( see also $[\mathbf{6}]$ ):

Definition 1.3. For an algebra $A$ in a Maltsev variety $\mathcal{V}$ and congruences $R$ and $S$ on $A$, the commutator $[R, S]$ is the smallest congruence on $A$ such that the function

$$
\left\{(x, y, z) \in A^{3} \mid(x, y) \in R \quad \text { and } \quad(y, z) \in S\right\} \mapsto A /[R, S]
$$

sending $(x, y, z)$ to the $[R, S]$-class of $p(x, y, z)$, is a homomorphism of algebras.

This definition does not depend on the choice of the Maltsev operation $p$ in the theory of the variety. Moreover, it does coincide with the classical definition introduced by Smith $[\mathbf{9}]$.

Remark 1.4. Looking at the Definition 1.3 and at Theorem 1.1.2, one can see, as remarked by Janelidze and Kelly [5], that the commutator $[R, S]$ of two congruences $R$ and $S$ on an algebra $A$ can be described as the subalgebra of $A \times A$ generated by all pairs $(u, v)$

$$
\begin{aligned}
& u=p\left(s\left(x_{1}, \ldots, x_{n}\right), s\left(y_{1}, \ldots, y_{n}\right), s\left(z_{1}, \ldots, z_{n}\right)\right) \\
& v=s\left(p\left(x_{1}, y_{1}, z_{1}\right), \ldots, p\left(x_{n}, y_{n}, z_{n}\right)\right)
\end{aligned}
$$

for some $n$-ary operator $s$ in a fixed signature of $\mathcal{V}$, for $p$ a Maltsev operation, and for elements $x_{1}, \ldots, x_{n}, y_{1}, \ldots, y_{n}, z_{1}, \ldots, z_{n}$ of $A$ having each $\left(x_{i}, y_{i}\right)$ in $R$ and each $\left(y_{i}, z_{i}\right)$ in $S$ (for $\left.i=1,2, \ldots n\right)$.

Here there are some basic properties of the commutator:

1. $[R, S]=[S, R]$;

2. $[R, S] \leqslant R \wedge S$;

3. $S \leqslant T \Rightarrow[R, S] \leqslant[R, T]$; 
4. $[R, S \vee T]=[R, S] \vee[R, T]$;

5. if $f: A \rightarrow B$ is a surjective homomorphism, then $f([R, S])=[f(R), f(S)]$,

where $f(R)$ denotes the image of the congruence $R$ along $f \times f: A \times A \rightarrow B \times B$. Remark that $f(R)$ actually is a congruence by Theorem 1.1.2. For any homomorphism $f: A \rightarrow B$, let $R[f]$ be its kernel congruence, i.e.:

$$
R[f]=\{(x, y) \in A \times A \mid f(x)=f(y)\} .
$$

We shall also use the property 5 of the commutator in the equivalent formulation

$$
5^{\prime} . f^{-1}[T, U]=R[f] \vee\left[f^{-1}(T), f^{-1}(U)\right]
$$

where $T$ and $U$ are congruences on $B, f^{-1}(T), f^{-1}(U)$ and $f^{-1}[T, U]$ are the respective inverse images.

By an extension $\left(A, f_{A}\right)$ of an algebra $A^{0}$ is simply meant a surjective homomorphism $f_{A}: A^{1} \rightarrow A^{0}$ in the variety $\mathcal{V}$. From now on we shall denote by $\Delta$ and $\nabla$ the smallest and the largest congruence on a fixed algebra, respectively.

Definition 1.5. An extension $\left(A, f_{A}\right)$ is central if $\left[R\left[f_{A}\right], \nabla\right]=\Delta$.

Let $\operatorname{Ext}(\mathcal{V})$ be the category of extensions in $\mathcal{V}$ : an arrow $\alpha$ from $\left(A, f_{A}\right)$ to $\left(B, f_{B}\right)$ in $\operatorname{Ext}(\mathcal{V})$ is given by a pair $\left(\alpha^{1}, \alpha^{0}\right)$ of homomorphisms in $\mathcal{V}$ such that $f_{B} \alpha^{1}=\alpha^{0} f_{A}$ :

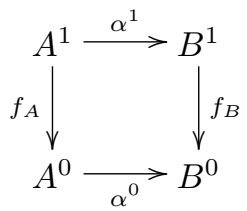

Let $\operatorname{Centr}(\mathcal{V})$ be the full subcategory of $\operatorname{Ext}(\mathcal{V})$ with objects all central extensions in $\mathcal{V}$. By using the properties of the commutator, one can see that $\operatorname{Centr}(\mathcal{V})$ is a reflective subcategory of $\operatorname{Ext}(\mathcal{V})$ (see [5], Remark 4.4), which will be displayed as

$$
\operatorname{Centr}(\mathcal{V}) \underset{U}{\frac{\perp}{L}} \operatorname{Ext}(\mathcal{V}) \text {. }
$$

The $\left(A, f_{A}\right)$-component of the unit of this adjunction is given by the arrow

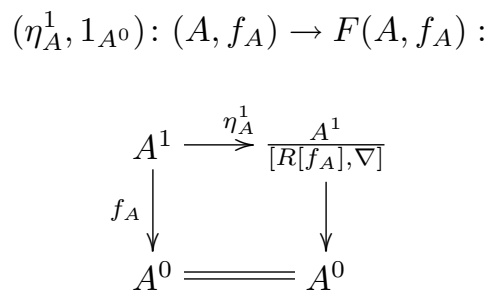

We shall often write $F\left(f_{A}\right): F\left(A^{1}\right) \rightarrow A^{0}$ for the induced right-hand vertical arrow in the diagram here above. 


\section{Galois structures}

In this section we recall the notion of Galois structure as defined by Janelidze in $[3]$ and $[4]$.

Let $\mathcal{C}$ be a category with pullbacks, and $\mathcal{E}$ a class of morphisms in $\mathcal{C}$ containing all isomorphisms, closed under composition and stable under pullbacks. For a fixed $(\mathcal{C}, \mathcal{E})$, we write $\mathcal{C} \downarrow B$ for the comma category over $B$, with $B$ an object in $\mathcal{C}$, and $\mathcal{E}(B)$ for its full subcategory whose objects are arrows $\sigma: C \rightarrow B$ in $\mathcal{E}$.

Given an arrow $\sigma: C \rightarrow B$ in $\mathcal{E}$ there is the composition functor $W^{\sigma}: \mathcal{E}(C) \rightarrow$ $\mathcal{E}(B)$ defined by $(A, \alpha) \mapsto(A, \sigma \alpha)$. Under our assumptions, $W^{\sigma}$ has a right adjoint $G^{\sigma}: \mathcal{E}(B) \rightarrow \mathcal{E}(C)$, the pullback functor along $\sigma$, defined by the assignment:

$$
(A, \alpha) \mapsto\left(C \times_{B} A, \pi_{C}\right) .
$$

Let us then recall from [4]:

Definition 2.1. A Galois structure $\Gamma$ consists of an adjunction

$$
\mathcal{X} \underset{U}{\stackrel{\frac{1}{u}}{<}} \mathcal{C}
$$

and classes $\mathcal{E}$ and $\mathcal{Z}$ of morphisms of $\mathcal{C}$ and $\mathcal{X}$, respectively, such that:

1. $(\mathcal{C}, \mathcal{E})$ satisfies the conditions above;

2. $(\mathcal{X}, \mathcal{Z})$ satisfies the same conditions;

3. $F(\mathcal{E}) \subset \mathcal{Z}$;

4. $U(\mathcal{Z}) \subset \mathcal{E}$;

5. the counit $\varepsilon$ is an isomorphism;

6. each component $\eta_{C}$ of the unit $\eta$ belongs to $\mathcal{E}$.

In this case we shall write $\Gamma=((\mathcal{X}, \mathcal{Z}),(\mathcal{C}, \mathcal{E}), U \vdash F)$. Given an object $C$ in $\mathcal{C}$, we call $F^{C}: \mathcal{C} \downarrow C \rightarrow \mathcal{X} \downarrow F(C)$ the functor induced by $F$, and $U^{C}: \mathcal{X} \downarrow F(C) \rightarrow \mathcal{C} \downarrow C$ its right adjoint. This latter is defined as follows: to any $(X, \varphi)$ in $\mathcal{X} \downarrow F(C)$, it associates $\left(C \times_{U F(C)} U(X), \pi_{C}\right)$ in $\mathcal{C} \downarrow C$, where the pullback is the following:

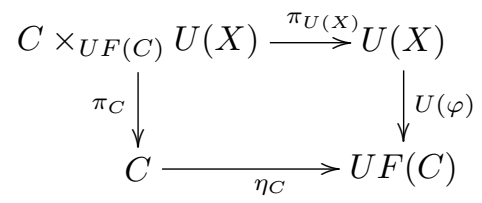

We denote by $F^{C, \Gamma}: \mathcal{E}(C) \rightarrow \mathcal{Z}(F(C))$ and $U^{C, \Gamma}: \mathcal{Z}(F(C)) \rightarrow \mathcal{E}(C)$ the functors induced by $F^{C}$ and $U^{C}$, respectively. 
Definition 2.2. Let $(A, \alpha)$ and $(C, \sigma)$ be two objects in $\mathcal{E}(B)$. We say that $(A, \alpha)$ is split over $(C, \sigma)$ with respect to $\Gamma$ if the canonical commutative diagram

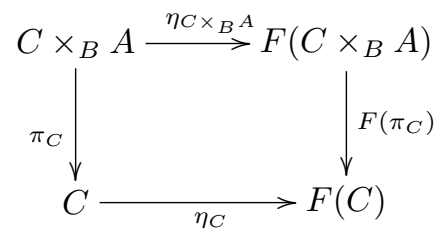

is a pullback.

Definition 2.3. Let $(A, \alpha)$ be in $\mathcal{E}(B) .(A, \alpha)$ is a $\Gamma$-covering if there exists a $(C, \sigma)$ in $\mathcal{E}(B)$ satisfying the following conditions:

1. $(A, \alpha)$ is split over $(C, \sigma)$ with respect to $\Gamma$;

2. the counit $\varepsilon^{C, \Gamma}: F^{C, \Gamma} U^{C, \Gamma} \rightarrow 1_{\mathcal{Z}(F(C))}$ is an isomorphism;

3. the functor $G^{\sigma}: \mathcal{E}(B) \rightarrow \mathcal{E}(C)$ is monadic.

The full subcategory of $\mathcal{E}(B)$ with objects all $\Gamma$-coverings is denoted by $\operatorname{Spl}(\Gamma, B)$.

\section{Galois structure of central extensions}

In this section we are first going to show that, in any Maltsev variety $\mathcal{V}$, the adjunction

$$
\operatorname{Centr}(\mathcal{V}) \underset{U}{\stackrel{F}{\leftrightarrows}} \operatorname{Ext}(\mathcal{V})
$$

gives rise to a Galois structure.

Let us first define the following class $\mathcal{E}$ of morphisms in $\operatorname{Ext}(\mathcal{V})$ : an arrow $\left(\alpha^{1}, \alpha^{0}\right):\left(A, f_{A}\right) \rightarrow\left(B, f_{B}\right)$

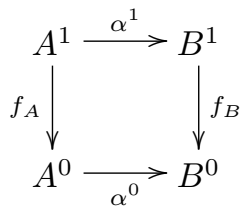

belongs to $\mathcal{E}$ if:

1. $\alpha^{1}$ and $\alpha^{0}$ are surjective homomorphisms in $\mathcal{V}$;

2. the canonical morphism $\left\langle f_{A}, \alpha^{1}\right\rangle: A^{1} \rightarrow A^{0} \times_{B^{0}} B^{1}$ to the pullback is surjective in $\mathcal{V}$.

We denote by $\mathcal{Z}$ the class of morphisms in $\operatorname{Centr}(\mathcal{V})$ satisfying the same conditions as the class $\mathcal{E}$ above.

Theorem 3.1. $((\operatorname{Centr}(\mathcal{V}), \mathcal{Z}),(\operatorname{Ext}(\mathcal{V}), \mathcal{E}), U \vdash F)$ is a Galois structure.

Proof. The forgetful functor $U: \operatorname{Centr}(\mathcal{V}) \rightarrow \operatorname{Ext}(\mathcal{V})$ has a left adjoint $F: \operatorname{Ext}(\mathcal{V}) \rightarrow$ $\operatorname{Centr}(\mathcal{V})$, as we recalled at the end of the first section. The category $\operatorname{Ext}(\mathcal{V})$ has 
pullbacks: indeed, given two arrows $\left(\alpha^{1}, \alpha^{0}\right):\left(A, f_{A}\right) \rightarrow\left(B, f_{B}\right)$ and $\left(\sigma^{1}, \sigma^{0}\right):\left(C, f_{C}\right) \rightarrow\left(B, f_{B}\right)$ in $\operatorname{Ext}(\mathcal{V})$, consider the following diagram

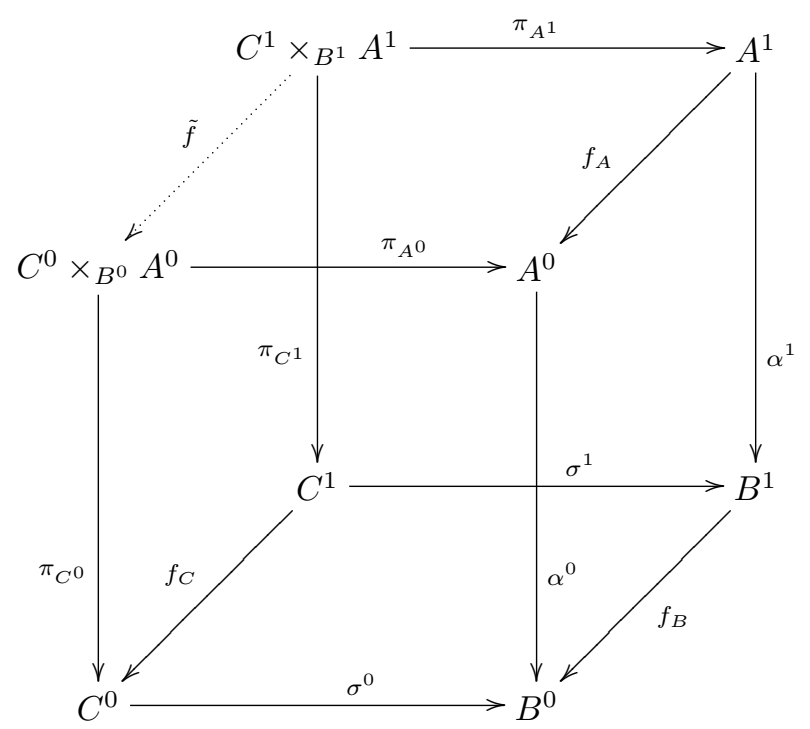

where the front and the back squares are pullbacks in $\mathcal{V}$. One can factorize the arrow $\tilde{f}: C^{1} \times_{B^{1}} A^{1} \rightarrow C^{0} \times{ }_{B^{0}} A^{0}$ as a surjective homomorphism $f: C^{1} \times{ }_{B^{1}} A^{1} \rightarrow$ $I$ followed by an injective homomorphism. The pullback of $\left(\alpha^{1}, \alpha^{0}\right):\left(A, f_{A}\right) \rightarrow$ $\left(B, f_{B}\right)$ and $\left(\sigma^{1}, \sigma^{0}\right):\left(C, f_{C}\right) \rightarrow\left(B, f_{B}\right)$ is given by the extension $f$. Remark that, when at least one of the arrows $\left(\alpha^{1}, \alpha^{0}\right)$ and $\left(\sigma^{1}, \sigma^{0}\right)$ is in $\mathcal{E}$, then $\tilde{f}=f$. The pullbacks also exist in $\operatorname{Centr}(\mathcal{V})$, and they are constructed in the same way.

One can check that both classes $\mathcal{Z}$ and $\mathcal{E}$ contain all the isomorphisms, are stable under composition and under pullbacks; moreover, $F(\mathcal{E}) \subset \mathcal{Z}$ and $U(\mathcal{Z}) \subset \mathcal{E}$. The counit $\varepsilon$ is an isomorphism because $\operatorname{Centr}(\mathcal{V})$ is a full subcategory of $\operatorname{Ext}(\mathcal{V})$. The $\left(A, f_{A}\right)$-component of the unit of the adjunction $U \vdash F$ clearly belongs to $\mathcal{E}$, as one can see by looking at the following diagram:

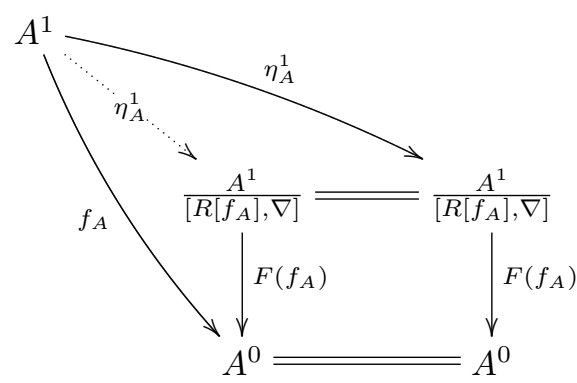

Convention. From now on, we shall only consider the special Galois structure

$$
((\operatorname{Centr}(\mathcal{V}), \mathcal{Z}),(\operatorname{Ext}(\mathcal{V}), \mathcal{E}), U \vdash F)
$$

in Theorem 3.1. 
With the same notations as in Section (2), we have the following

Lemma 3.2. Let $(C, \sigma)$ be any object in $\mathcal{E}(B)$. Then:

1. the counit $\varepsilon^{C, \Gamma}: F^{C, \Gamma} U^{C, \Gamma} \rightarrow 1_{\mathcal{Z}(F(C))}$ of the adjunction $U^{C, \Gamma} \vdash F^{C, \Gamma}$ is an isomorphism;

2. the pullback functor $G^{\sigma}: \mathcal{E}(B) \rightarrow \mathcal{E}(C)$ is monadic.

Proof. (1) Let $(X, \varphi)$ be an object in $\mathcal{Z}(F(C))$, and let $\left(C \times_{F(C)} X, f\right)$ be the extension obtained by taking the pullback in $\operatorname{Ext}(\mathcal{V})$ of $\left(\varphi^{1}, \varphi^{0}\right)$ and the $\left(C, f_{C}\right)$ component $\left(\eta_{C}^{1}, 1_{C^{0}}\right)$ of the unit of the adjunction $U \vdash F$.

The $(X, \varphi)$-component of the counit $\varepsilon^{C, \Gamma}$ is a pair of arrows $\left(\varepsilon_{\varphi^{1}}^{C, \Gamma}, 1_{X^{0}}\right)$. In order to prove that $\varepsilon_{\varphi^{1}}^{C, \Gamma}$ is an isomorphism, consider the following diagram

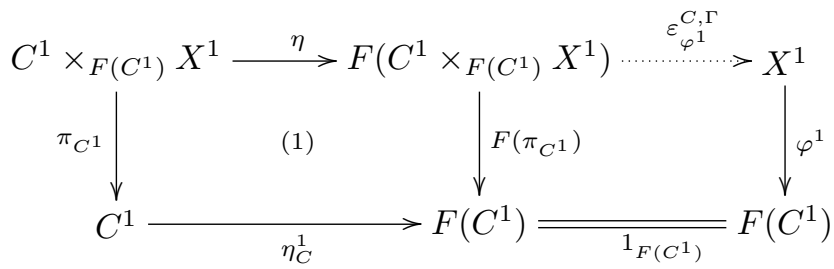

where $\eta=\eta_{C \times_{F(C)} X}^{1}$. Since $\varepsilon_{\varphi^{1}}^{C, \Gamma} \eta=\pi_{X^{1}}$, it follows that $\varepsilon_{\varphi^{1}}^{C, \Gamma}$ is surjective. In order to prove that $\varepsilon_{\varphi^{1}}^{C, \Gamma}$ is an isomorphism, it is sufficient to show that the kernel congruences $R[\eta]$ and $R\left[\pi_{X^{1}}\right]$ are equal. Of course, $R[\eta] \leqslant R\left[\varepsilon_{\varphi^{1}}^{C, \Gamma} \eta\right]=R\left[\pi_{X^{1}}\right]$. To prove the converse inclusion, first observe that $R\left[\pi_{C^{1}}\right] \wedge R\left[\pi_{X^{1}}\right]=\Delta$ because $\pi_{C^{1}}$ and $\pi_{X^{1}}$ are the projections of a pullback. Then the inequality $R[\eta] \leqslant R\left[\pi_{X^{1}}\right]$ together with the modularity of the lattice of congruences in $\mathcal{V}$ gives:

$$
R[\eta]=R[\eta] \vee\left(R\left[\pi_{C^{1}}\right] \wedge R\left[\pi_{X^{1}}\right]\right)=\left(R[\eta] \vee R\left[\pi_{C^{1}}\right]\right) \wedge R\left[\pi_{X^{1}}\right]=R\left[\pi_{X^{1}}\right] .
$$

The last equality follows from the fact that the square (1) is a pushout in $\mathcal{V}$ (which can be deduced from the property 5 of the commutator in a Maltsev variety), so that

$$
R\left[\pi_{X^{1}}\right] \leqslant R\left[\varphi^{1} \pi_{X^{1}}\right]=R\left[\eta_{C}^{1} \pi_{C^{1}}\right]=R[\eta] \vee R\left[\pi_{C^{1}}\right] .
$$

(2) For $i=0,1$ the pullback functor $G^{\sigma^{i}}: \mathcal{V} \downarrow B^{i} \rightarrow \mathcal{V} \downarrow C^{i}$ is monadic [7]. From this it follows easily that the pullback functor $G^{\sigma}: \mathcal{E}(B) \rightarrow \mathcal{E}(C)$ along $\sigma=\left(\sigma^{1}, \sigma^{0}\right)$ is also monadic.

Thanks to the previous Lemma, when $\Gamma=((\operatorname{Centr}(\mathcal{V}), \mathcal{Z}),(\operatorname{Ext}(\mathcal{V}), \mathcal{E}), U \vdash F)$, the Definition 2.3 of a $\Gamma$-covering can be simplified:

Definition 3.3. Let $(A, \alpha)$ in $\mathcal{E}(B)$ :

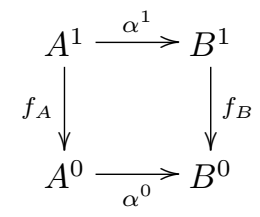


$(A, \alpha)$ is a $\Gamma$-covering if there exists a $(C, \sigma)$ in $\mathcal{E}(B)$ such that

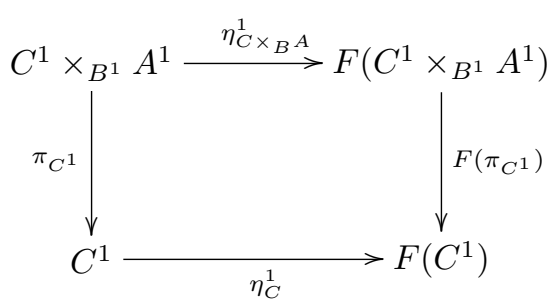

is a pullback in $\mathcal{V}$.

\section{Double Central Extensions}

In this section we define double central extensions and prove that any $\Gamma$-covering, as defined in the previous section, is a double central extension.

Definition 4.1. Let $\mathcal{V}$ be a variety. A double extension $(A, \alpha)$ of $\left(B, f_{B}\right)$ is an element in $\mathcal{E}(B)$. This means that in the commutative square

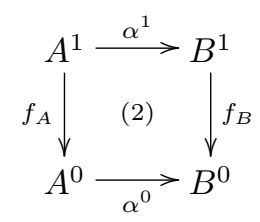

the homomorphisms $\alpha^{1}$ and $\alpha^{0}$ are surjective, as well as the canonical morphism $\left\langle f_{A}, \alpha^{1}\right\rangle: A^{1} \rightarrow A^{0} \times{ }_{B^{0}} B^{1}$ to the pullback.

Definition 4.2. Let $\mathcal{V}$ be a Maltsev variety. A double extension $(A, \alpha)$ of $\left(B, f_{B}\right)$ is called a double central extension if the following conditions are satisfied:

1. $\left[R\left[\alpha^{1}\right], R\left[f_{A}\right]\right]=\Delta$;

2. $\left[R\left[\alpha^{1}\right] \wedge R\left[f_{A}\right], \nabla\right]=\Delta$.

Remark 4.3. Let us first notice that a double extension (2) as in Definition 4.1 is central whenever the arrow $f_{A}$ is a central extension (in the sense of Definition 1.5). Consequently, in order to prove that any $\Gamma$-covering is a double central extension it 
suffices to show that in any pullback diagram:

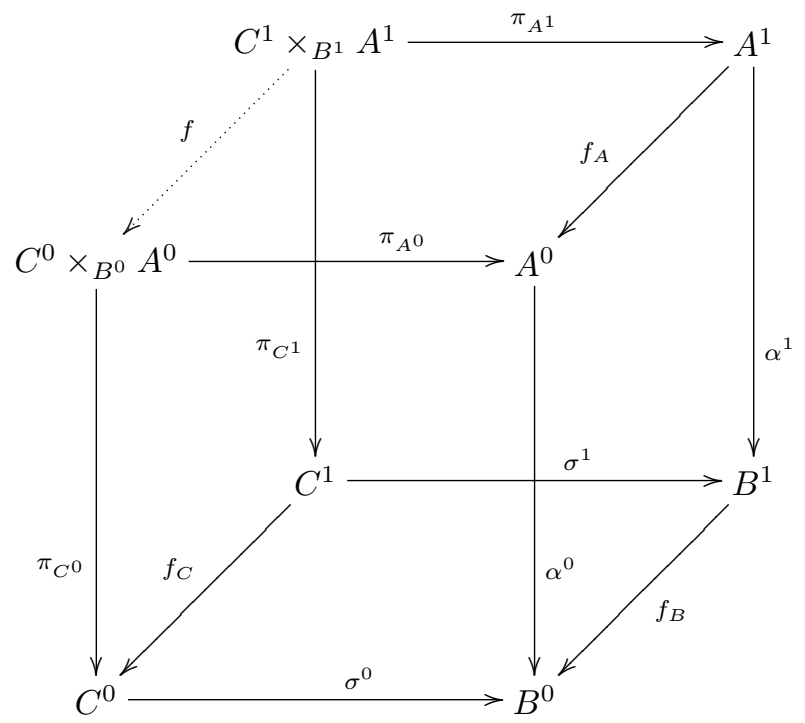

in $\operatorname{Ext}(\mathcal{V})$ with $(C, \sigma)$ in $\mathcal{E}(B)$, the double extension $(A, \alpha)$ is central if and only if the double extension $\left(C \times_{B} A, \pi_{C}\right)$ is central. This is exactly what we are going to prove in the rest of this section.

We begin with the following observation:

Lemma 4.4. With the same notations as in diagram (3):

$$
\pi_{A^{1}}\left(R\left[\pi_{C^{1}}\right] \wedge R[f]\right)=R\left[\alpha^{1}\right] \wedge R\left[f_{A}\right] .
$$

Proof. One always has that

$$
\pi_{A^{1}}\left(R\left[\pi_{C^{1}}\right] \wedge R[f]\right) \leqslant \pi_{A^{1}}\left(R\left[\pi_{C^{1}}\right]\right) \wedge \pi_{A^{1}}(R[f])=R\left[\alpha^{1}\right] \wedge R\left[f_{A}\right],
$$

where the equality follows from the fact that $\sigma$ is an arrow in $\mathcal{E}$. Conversely, let $\left(a_{1}, a_{2}\right)$ be in $R\left[\alpha^{1}\right] \wedge R\left[f_{A}\right]$, and let us call $b=\alpha^{1}\left(a_{1}\right)=\alpha^{1}\left(a_{2}\right)$. There exists a $c_{0}$ in $C^{0}$ such that $\sigma^{0}\left(c_{0}\right)=f_{B}(b)$. Once again, since $\sigma$ is in $\mathcal{E}$, there exists $c_{1}$ in $C^{1}$ such that $\sigma^{1}\left(c_{1}\right)=b$ and $f_{C}\left(c_{1}\right)=c_{0}$. Therefore the element $\left(\left(c_{1}, a_{1}\right),\left(c_{1}, a_{2}\right)\right)$ is in $R\left[\pi_{C^{1}}\right] \wedge R[f]$, and $R\left[\alpha^{1}\right] \wedge R\left[f_{A}\right] \leqslant \pi_{A^{1}}\left(R\left[\pi_{C^{1}}\right] \wedge R[f]\right)$, as desired.

Proposition 4.5. With the same notations as in diagram $(3)$ : if $\left(C \times_{B} A, \pi_{C}\right)$ is a double central extension, then $(A, \alpha)$ is a double central extension.

Proof. The first property in the Definition 4.2 of double central extension follows from the property 5 of the commutator: indeed, from $\left[R\left[\pi_{C^{1}}\right], R[f]\right]=\Delta$ one has that

$$
\left[R\left[\alpha^{1}\right], R\left[f_{A}\right]\right]=\left[\pi_{A^{1}}\left(R\left[\pi_{C^{1}}\right]\right), \pi_{A^{1}}(R[f])\right]=\pi_{A^{1}}\left(\left[R\left[\pi_{C^{1}}\right], R[f]\right]\right)=\Delta .
$$


The previous Lemma and the condition $\left[R\left[\pi_{C^{1}}\right] \wedge R[f], \nabla\right]=\Delta$, together with the property 5 of the commutator, imply that:

$$
\left[R\left[\alpha^{1}\right] \wedge R\left[f_{A}\right], \nabla\right]=\left[\pi_{A^{1}}\left(R\left[\pi_{C^{1}}\right] \wedge R[f]\right), \nabla\right]=\pi_{A^{1}}\left(\left[R\left[\pi_{C^{1}}\right] \wedge R[f], \nabla\right]\right)=\Delta .
$$

Lemma 4.6. Let $f: A \rightarrow B$ be a surjective homomorphism, and let $R$ and $S$ be congruences on $A$ such that $S \wedge R[f]=\Delta$. If $[f(R), f(S)]=\Delta$, then $[R, S]=\Delta$.

Proof. We first show that, under our assumptions, $[R, S] \leqslant R[f]$. By property $5^{\prime}$ in Section 1 we have:

$$
R[f] \vee\left[f^{-1}(f(R)), f^{-1}(f(S))\right]=f^{-1}([f(R), f(S)])=f^{-1}(\Delta)=R[f] .
$$

Therefore, by property 3 of the commutator:

$$
R[f] \geqslant\left[f^{-1}(f(R)), f^{-1}(f(S))\right] \geqslant[R, S] .
$$

By the property 2 of the commutator, we have that $[R, S] \leqslant R \wedge S$, thus:

$$
[R, S] \leqslant(R \wedge S) \wedge R[f]=R \wedge(S \wedge R[f])=\Delta .
$$

Proposition 4.7. With the same notations as in diagram (3): if $(A, \alpha)$ is a double central extension, then $\left(C \times_{B} A, \pi_{C}\right)$ is a double central extension.

Proof. By assumption $\left[R\left[\alpha^{1}\right], R\left[f_{A}\right]\right]=\Delta$ and $\left[R\left[\alpha^{1}\right] \wedge R\left[f_{A}\right], \nabla\right]=\Delta$. Moreover, since

$$
R\left[\pi_{C^{1}}\right] \wedge R\left[\pi_{A^{1}}\right]=\Delta,
$$

and

$$
\left[\pi_{A^{1}}\left(R\left[\pi_{C^{1}}\right]\right), \pi_{A^{1}}(R[f])\right]=\left[R\left[\alpha^{1}\right], R\left[f_{A}\right]\right]=\Delta,
$$

the previous Lemma implies that $\left[R\left[\pi_{C^{1}}\right], R[f]\right]=\Delta$. Similarly,

$$
\left[\pi_{A^{1}}\left(R\left[\pi_{C^{1}}\right] \wedge R[f]\right), \pi_{A^{1}}(\nabla)\right]=\left[R\left[\alpha^{1}\right] \wedge R\left[f_{A}\right], \nabla\right]=\Delta .
$$

Again by applying the previous Lemma, it follows that:

$$
\left[R\left[\pi_{C^{1}}\right] \wedge R[f], \nabla\right]=\Delta
$$

Theorem 4.8. Any $\Gamma$-covering is a double central extension.

\section{The characterization of $\Gamma$-coverings}

We are now going to show that the $\Gamma$-coverings are exactly the double central extensions.

Theorem 5.1. Any double central extension is a $\Gamma$-covering. 
Proof. Let $(A, \alpha)$ be a double central extension. Consider the pullback of $(A, \alpha)$ along itself:

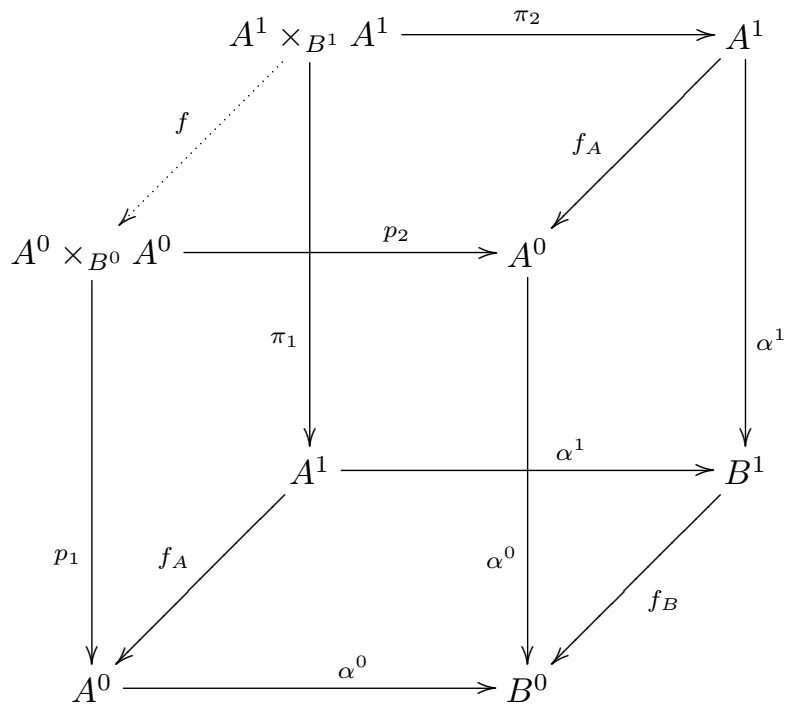

Remark that by Proposition 4.7 the left-hand double extension in the cube above is central, that is

$$
\begin{gathered}
{\left[R\left[\pi_{1}\right], R[f]\right]=\Delta,} \\
{\left[R\left[\pi_{1}\right] \wedge R[f], \nabla\right]=\Delta .}
\end{gathered}
$$

In order to prove that $(A, \alpha)$ is a $\Gamma$-covering, we must show that the canonical commutative square

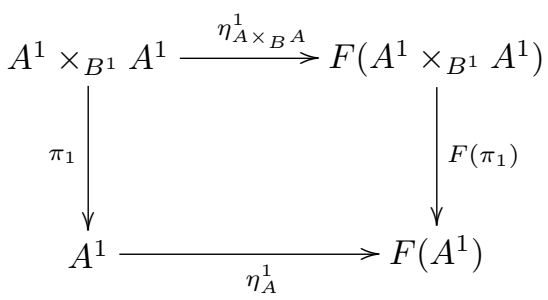

is a pullback. Clearly, this square is a pushout of surjective homomorphisms because $\pi_{1}$ and $F\left(\pi_{1}\right)$ are naturally split. Since $\mathcal{V}$ is a Maltsev variety, by Proposition 1.2 the comparison arrow $w: A^{1} \times_{B^{1}} A^{1} \rightarrow A^{1} \times_{F\left(A^{1}\right)} F\left(A^{1} \times_{B^{1}} A^{1}\right)$ is a surjective homomorphism. Consequently, to prove that it is a pullback it suffices to show that:

$$
R\left[\pi_{1}\right] \wedge R\left[\eta_{A \times{ }_{B} A}^{1}\right]=\Delta .
$$

By definition of the functor $F$, this means that $(A, \alpha)$ is a $\Gamma$-covering if and only if

$$
R\left[\pi_{1}\right] \wedge[R[f], \nabla]=\Delta .
$$


The claim of the Theorem reduces then to the implication $(1)+(2) \Rightarrow(3)$.

Let us denote by $\delta: A^{1} \rightarrow A^{1} \times{ }_{B^{1}} A^{1}$ the unique homomorphism such that $\pi_{1} \delta=1_{A^{1}}=\pi_{2} \delta$.

By Remark 1.4 the condition (1) says that, for any $n$-ary term $s$ and any Maltsev operation $p$ the elements

$$
\begin{aligned}
& u=p\left(s\left(x_{1}, \ldots, x_{n}\right), s\left(y_{1}, \ldots, y_{n}\right), s\left(z_{1}, \ldots, z_{n}\right)\right) \\
& v=s\left(p\left(x_{1}, y_{1}, z_{1}\right), \ldots, p\left(x_{n}, y_{n}, z_{n}\right)\right)
\end{aligned}
$$

are equal whenever $\pi_{1}\left(x_{i}\right)=\pi_{1}\left(y_{i}\right)$ and $f\left(y_{i}\right)=f\left(z_{i}\right)$ for $i=1, \ldots, n$.

In the same way, the condition (2) means that $u=v$ whenever $\pi_{1}\left(x_{i}\right)=\pi_{1}\left(y_{i}\right)$ and $f\left(x_{i}\right)=f\left(y_{i}\right)$ for $i=1, \ldots, n$.

Let us define a function $+:\left(A^{1} \times_{B^{1}} A^{1}\right) \times\left(A^{1} \times_{B^{1}} A^{1}\right) \rightarrow A^{1} \times_{B^{1}} A^{1}$ by setting

$$
u+v=p\left(u, \delta \pi_{1}(u), \delta \pi_{1}(v)\right) .
$$

If $(u, v)$ belong to $R\left[\pi_{1}\right]$, we have:

$$
u+v=p\left(u, \delta \pi_{1}(u), \delta \pi_{1}(v)\right)=p\left(u, \delta \pi_{1}(u), \delta \pi_{1}(u)\right)=u .
$$

To prove (3), it will be sufficient to show that $u+v=v$ for each $(u, v)$ in $[R[f], \nabla]$.

The proof will consist of two steps. In the first step we shall prove that $u+v=v$ for any "generator" $(u, v)$ of the commutator $[R[f], \nabla]$. In the second step we shall extend the equality $u+v=v$ to any $(u, v)$ in $[R[f], \nabla]$.

\section{FIRST STEP:}

Let $(u, v)$ be a generator of the commutator $[R[f], \nabla]$, i.e. $u$ and $v$ can be written as in (4) and (5) with $f\left(x_{i}\right)=f\left(y_{i}\right)$ for $i=1, \ldots, n$.

Let us calculate $u+v$ :

$$
\begin{aligned}
u+v= & u+p\left(s\left(x_{1}, \ldots, x_{n}\right), s\left(x_{1}, \ldots, x_{n}\right), v\right) \\
= & p\left(u, \delta \pi_{1}(u), \delta \pi_{1} p\left(s\left(x_{1}, \ldots, x_{n}\right), s\left(x_{1}, \ldots, x_{n}\right), v\right)\right) \\
= & p\left(p\left(s\left(x_{1}, \ldots, x_{n}\right), s\left(y_{1}, \ldots, y_{n}\right), s\left(z_{1}, \ldots, z_{n}\right)\right),\right. \\
& \delta \pi_{1} p\left(s\left(x_{1}, \ldots, x_{n}\right), s\left(y_{1}, \ldots, y_{n}\right), s\left(z_{1}, \ldots, z_{n}\right)\right), \\
& \left.\delta \pi_{1} p\left(s\left(x_{1}, \ldots, x_{n}\right), s\left(x_{1}, \ldots, x_{n}\right), v\right)\right) .
\end{aligned}
$$

By taking into account the fact that $\delta \pi_{1}$ is a homomorphism, this becomes:

$$
\begin{aligned}
=p & \left(p\left(s\left(x_{1}, \ldots, x_{n}\right), s\left(y_{1}, \ldots, y_{n}\right), s\left(z_{1}, \ldots, z_{n}\right)\right),\right. \\
& p\left(\delta \pi_{1} s\left(x_{1}, \ldots, x_{n}\right), \delta \pi_{1} s\left(y_{1}, \ldots, y_{n}\right), \delta \pi_{1} s\left(z_{1}, \ldots, z_{n}\right)\right), \\
& \left.p\left(\delta \pi_{1} s\left(x_{1}, \ldots, x_{n}\right), \delta \pi_{1} s\left(x_{1}, \ldots, x_{n}\right), \delta \pi_{1}(v)\right)\right) .
\end{aligned}
$$

Here the condition (1) can be applied, giving:

$$
\begin{array}{rl}
(*)=p & p\left(s\left(x_{1}, \ldots, x_{n}\right), \delta \pi_{1} s\left(x_{1}, \ldots, x_{n}\right), \delta \pi_{1} s\left(x_{1}, \ldots, x_{n}\right)\right), \\
& p\left(s\left(y_{1}, \ldots, y_{n}\right), \delta \pi_{1} s\left(y_{1}, \ldots, y_{n}\right), \delta \pi_{1} s\left(x_{1}, \ldots, x_{n}\right)\right), \\
& \left.p\left(s\left(z_{1}, \ldots, z_{n}\right), \delta \pi_{1} s\left(z_{1}, \ldots, z_{n}\right), \delta \pi_{1}(v)\right)\right) .
\end{array}
$$

We now analyse separately the three components in the last expression $(*)$. 
First,

$$
p\left(s\left(x_{1}, \ldots, x_{n}\right), \delta \pi_{1} s\left(x_{1}, \ldots, x_{n}\right), \delta \pi_{1} s\left(x_{1}, \ldots, x_{n}\right)\right)=s\left(x_{1}, \ldots, x_{n}\right)
$$

with $x_{i}^{\prime}=x_{i}$. Secondly,

$$
=s\left(x_{1}^{\prime}, \ldots, x_{n}^{\prime}\right)
$$

$$
\begin{aligned}
& p\left(s\left(y_{1}, \ldots, y_{n}\right), \delta \pi_{1} s\left(y_{1}, \ldots, y_{n}\right), \delta \pi_{1} s\left(x_{1}, \ldots, x_{n}\right)\right) \\
= & p\left(s\left(y_{1}, \ldots, y_{n}\right), s\left(\delta \pi_{1}\left(y_{1}\right), \ldots, \delta \pi_{1}\left(y_{n}\right)\right), s\left(\delta \pi_{1}\left(x_{1}\right), \ldots, \delta \pi_{1}\left(x_{n}\right)\right)\right) \\
(6)= & s\left(p\left(y_{1}, \delta \pi_{1}\left(y_{1}\right), \delta \pi_{1}\left(x_{1}\right)\right), \ldots, p\left(y_{n}, \delta \pi_{1}\left(y_{n}\right), \delta \pi_{1}\left(x_{n}\right)\right)\right) \\
= & s\left(y_{1}^{\prime}, \ldots, y_{n}^{\prime}\right)
\end{aligned}
$$

with $y_{i}^{\prime}=p\left(y_{i}, \delta \pi_{1}\left(y_{i}\right), \delta \pi_{1}\left(x_{i}\right)\right)$. The equality (6) follows from the condition (1).

Thirdly,

$$
\begin{aligned}
& p\left(s\left(z_{1}, \ldots, z_{n}\right), \delta \pi_{1} s\left(z_{1}, \ldots, z_{n}\right), \delta \pi_{1} s\left(p\left(x_{1}, y_{1}, z_{1}\right), \ldots, p\left(x_{n}, y_{n}, z_{n}\right)\right)\right) \\
= & p\left(s\left(z_{1}, \ldots, z_{n}\right), s\left(\delta \pi_{1}\left(z_{1}\right), \ldots, \delta \pi_{1}\left(z_{n}\right)\right), s\left(\delta \pi_{1} p\left(x_{1}, y_{1}, z_{1}\right), \ldots, \delta \pi_{1} p\left(x_{n}, y_{n}, z_{n}\right)\right)\right) \\
(7)= & s\left(p\left(z_{1}, \delta \pi_{1}\left(z_{1}\right), \delta \pi_{1} p\left(x_{1}, y_{1}, z_{1}\right)\right), \ldots, p\left(z_{n}, \delta \pi_{1}\left(z_{n}\right), \delta \pi_{1} p\left(x_{n}, y_{n}, z_{n}\right)\right)\right) \\
= & s\left(z_{1}^{\prime}, \ldots, z_{n}^{\prime}\right)
\end{aligned}
$$

with $z_{i}^{\prime}=p\left(z_{i}, \delta \pi_{1}\left(z_{i}\right), \delta \pi_{1} p\left(x_{i}, y_{i}, z_{i}\right)\right)$. The equality (7) follows from the condition (1).

Let us now go back to the expression $(*)$ :

$$
\begin{aligned}
& p\left(p\left(s\left(x_{1}, \ldots, x_{n}\right), \delta \pi_{1} s\left(x_{1}, \ldots, x_{n}\right), \delta \pi_{1} s\left(x_{1}, \ldots, x_{n}\right)\right),\right. \\
& p\left(s\left(y_{1}, \ldots, y_{n}\right), \delta \pi_{1} s\left(y_{1}, \ldots, y_{n}\right), \delta \pi_{1} s\left(x_{1}, \ldots, x_{n}\right)\right), \\
& \left.p\left(s\left(z_{1}, \ldots, z_{n}\right), \delta \pi_{1} s\left(z_{1}, \ldots, z_{n}\right), \delta \pi_{1}(v)\right)\right) \\
= & p\left(s\left(x_{1}^{\prime}, \ldots, x_{n}^{\prime}\right), s\left(y_{1}^{\prime}, \ldots, y_{n}^{\prime}\right), s\left(z_{1}^{\prime}, \ldots, z_{n}^{\prime}\right)\right) \\
(8)= & s\left(p\left(x_{1}^{\prime}, y_{1}^{\prime}, z_{1}^{\prime}\right), \ldots, p\left(x_{n}^{\prime}, y_{n}^{\prime}, z_{n}^{\prime}\right)\right) \\
(9)= & s\left(p\left(x_{1}, y_{1}, z_{1}\right), \ldots, p\left(x_{n}, y_{n}, z_{n}\right)\right) \\
= & v .
\end{aligned}
$$

The equality (8) follows from the condition (2). On the other hand, the equality (9) depends on the fact that $p\left(x_{i}^{\prime}, y_{i}^{\prime}, z_{i}^{\prime}\right)=p\left(x_{i}, y_{i}, z_{i}\right)$ for each $i=1, \ldots, n$. Indeed, by definition of + one has:

$$
p\left(x_{i}, y_{i}, z_{i}\right)+p\left(x_{i}, y_{i}, z_{i}\right)=p\left(x_{i}, y_{i}, z_{i}\right) .
$$

Consequently,

$$
\begin{aligned}
& p\left(x_{i}, y_{i}, z_{i}\right) \\
= & p\left(x_{i}, y_{i}, z_{i}\right)+p\left(x_{i}, y_{i}, z_{i}\right) \\
= & p\left(x_{i}, y_{i}, z_{i}\right)+p\left(x_{i}, x_{i}, p\left(x_{i}, y_{i}, z_{i}\right)\right) \\
= & p\left(p\left(x_{i}, y_{i}, z_{i}\right), \delta \pi_{1} p\left(x_{i}, y_{i}, z_{i}\right), \delta \pi_{1} p\left(x_{i}, x_{i}, p\left(x_{i}, y_{i}, z_{i}\right)\right)\right) \\
= & p\left(p\left(x_{i}, y_{i}, z_{i}\right), p\left(\delta \pi_{1}\left(x_{i}\right), \delta \pi_{1}\left(y_{i}\right), \delta \pi_{1}\left(z_{i}\right)\right), p\left(\delta \pi_{1}\left(x_{i}\right), \delta \pi_{1}\left(x_{i}\right), \delta \pi_{1} p\left(x_{i}, y_{i}, z_{i}\right)\right)\right) \\
(10)= & p\left(p\left(x_{i}, \delta \pi_{1}\left(x_{i}\right), \delta \pi_{1}\left(x_{i}\right)\right), p\left(y_{i}, \delta \pi_{1}\left(y_{i}\right), \delta \pi_{1}\left(x_{i}\right)\right), p\left(z_{i}, \delta \pi_{1}\left(z_{i}\right), \delta \pi_{1} p\left(x_{i}, y_{i}, z_{i}\right)\right)\right) \\
= & p\left(x_{1}^{\prime}, y_{1}^{\prime}, z_{1}^{\prime}\right)
\end{aligned}
$$

where (10) is a consequence of the condition (1). 


\section{SECOND STEP:}

Let $X$ be the set of generators of $[R[f], \nabla]$, considered as a subalgebra of $\left(A^{1} \times_{B^{1}} A^{1}\right) \times\left(A^{1} \times_{B^{1}} A^{1}\right)$. The congruence $[R[f], \nabla]$ is then given by

$$
[R[f], \nabla]=\bigcup_{m \in \mathbb{N}} E^{m}(X),
$$

where $E^{0}(X)=X, E(X)=X \cup\left\{s\left(\left(u_{1}, v_{1}\right), \ldots,\left(u_{n}, v_{n}\right)\right) \mid s \in \Omega,\left(u_{i}, v_{i}\right) \in X\right\}$, and $E^{m+1}(X)=E\left(E^{m}(X)\right)$. We shall use induction on $m$.

The case $m=0$ is exactly given by the first step above. Let us then assume that $u+v=v$ for any $(u, v)$ in $E^{m}(X)$. If we take $(u, v)$ in $E^{m+1}(X)$, either it is in $E^{m}(X)$, or it can be written as

$$
(u, v)=\left(s\left(u_{1}, \ldots, u_{n}\right), s\left(v_{1}, \ldots, v_{n}\right)\right)
$$

with $\left(u_{i}, v_{i}\right)$ in $E^{m}(X)$, and $s$ in $\Omega$.

$$
\begin{aligned}
u+v & =p\left(s\left(u_{1}, \ldots, u_{n}\right), \delta \pi_{1} s\left(u_{1}, \ldots, u_{n}\right), \delta \pi_{1} s\left(v_{1}, \ldots, v_{n}\right)\right) \\
& =p\left(s\left(u_{1}, \ldots, u_{n}\right), s\left(\delta \pi_{1}\left(u_{1}\right), \ldots, \delta \pi_{1}\left(u_{n}\right)\right), s\left(\delta \pi_{1}\left(v_{1}\right), \ldots, \delta \pi_{1}\left(v_{n}\right)\right)\right) \\
(11) & =s\left(p\left(u_{1}, \delta \pi_{1}\left(u_{1}\right), \delta \pi_{1}\left(v_{1}\right)\right), \ldots, p\left(u_{n}, \delta \pi_{1}\left(u_{n}\right), \delta \pi_{1}\left(v_{n}\right)\right)\right) \\
& =s\left(u_{1}+v_{1}, \ldots, u_{n}+v_{n}\right) \\
(12) & =s\left(v_{1}, \ldots, v_{n}\right) \\
& =v
\end{aligned}
$$

The equality (11) follows from condition (1) (which applies since $[R[f], \nabla] \subseteq R[f]$ ), while (12) follows from the inductive assumption.

Corollary 5.2. In a Maltsev variety $\mathcal{V}$ a double extension is a $\Gamma$-covering if and only if it is a double central extension.

\section{References}

[1] R. Brown and G. Ellis, Hopf formulae for the higher homology of a group, Bull. London Math. Soc. 20, 1988, 124-128.

[2] A. Carboni, G.M. Kelly and M.C. Pedicchio, Some remarks on Maltsev and Goursat categories, Appl. Cat. Struct., 1, 1993, 385-421.

[3] G. Janelidze, Pure Galois theory in categories, J. Algebra 132, 1990, 270-286.

[4] G. Janelidze, What is a double central extension? (The question was asked by Ronald Brown), Cah. Topologie Géom. Différ. Catég. 32, No 3, 1991, 191-201.

[5] G. Janelidze and G.M. Kelly, Central extensions in Maltsev varieties, Theory Appl. Categ., 7, No 10, 2000, 219-226.

[6] G. Janelidze and M.C. Pedicchio, Pseudogroupoids and commutators, Theory Appl. Categ., 8, No 15, 2001, 408-456.

[7] G. Janelidze and W. Tholen, Facets of Descent I, Appl. Cat. Struct., 2, 1994, 245-281. 
[8] A.I. Mal'cev, On the general theory of algebraic systems, Mat. Sbornik N.S., 35, 1954, 3-20.

[9] J.D.H. Smith, Mal'cev Varieties, Lect. Notes Math. 554, Springer-Verlag, 1976.

This article may be accessed via WWW at http://www.rmi.acnet.ge/hha/ or by anonymous ftp at

ftp://ftp.rmi.acnet.ge/pub/hha/volumes/2004/n1a16/v6n1a16.(dvi,ps,pdf)

Marino Gran gran@lmpa.univ-littoral.fr

Université du Littoral Côte d'Opale, Laboratoire de Mathématiques Pures et Appliquées, Bt. H. Poincaré, 50 Rue F. Buisson BP 699,

62228 Calais, France.

Valentina Rossi rossi@dimi.uniud.it

Università degli Studi di Udine,

Dipartimento di Matematica e Informatica,

Via delle Scienze 206,

33100 , Udine, Italy. 